\title{
Research Framework for Emergency Rescue of Disasters and Crises
}

\author{
Zhi Zhang, Jianbo You, Qiaoying Li, Xuan Du \\ The Corps College of the Chinese Armed Police Force, Beijing 102202, China \\ E-mail:wjzhangzhi@139.com \\ Received 27 May 2012 \\ Accepted 12 October 2012
}

\begin{abstract}
The emergency rescue refers to all the artificial interventions that aim to reduce the occurrence of and damages by the emergencies directly or indirectly. Its role is to act immediately to prevent damage to life, property or environment. Emergency rescue is the critical and direct stage in the emergency management process. Disasters and crises have the nature of the sudden urgency, complexity, severity, and decide the characteristics of emergency rescue operations are urgent, difficult, dangerous and hard. The essay comes up with research framework of professional rescue: the emergency organization and command of multi-forces on strategy-battle-tactics level, the risk analysis of multi-disasters events and the risk management and control of rescue, the standardization of rescue skills and equipments and professional training, and the information platform combining application system, database and the preliminary analysis of main sources.
\end{abstract}

Keywords: Emergency rescue, Research framework, Command, Risk management

\section{Introduction}

After September 11 event in 2001, research in emergency rescue has attracted significant attention. The goal of research is to reduce the damages caused by emergencies and to sustain public safety. After the event of fight against SARS in 2003, China has started to build the national emergency management system in an all-round way, with "one plan and three mechanisms" (compilation and revise of emergency plan, enhancement the construction of emergency management system, mechanism, and law) as the core ${ }^{[1]}$. In the face of highly complex trend of emergency events in modern society after the Wenchuan earthquake in Sichuan Province in southwest China in 2008, the training of emergency rescue stuff has been given the priority. A magnitude 9.0 earthquake struck off the northeastern coast of Japan on March 11, 2011, in which 15,854 people were killed, and another 3271 people missing. The knock-on effect caused by the earthquake, tsunami and nuclear accident has increased the difficulty of the task of the emergency rescue.

However, it is somehow still difficult to provide a general view on the core scientific problems in the emergency rescue research due to its complexity.
One common understanding is that emergency rescue research should include risk analysis and assessment, emergency response, operations Command, rescue team organization and coordination, and recovery, etc, based on the development and changes of disasters and crises. Although there are large numbers of researchers and topics in emergency rescue, there still exist three questions: How to determine the research framework according to the characteristics of emergency rescue? How to combine emergency management and emergency rescue operations effectively? What are the main directions and their contents in the research framework?

The emergency and its response and management have typical distinction of complex systems just like the coupled human and natural systems ${ }^{[2]}$. It is a multi-subsystem, multi-level, multi-function system and the systems and factors involved are very often uncertain, non-linear, dynamic and open. Thus, neither reductionism nor system approach is sufficient ${ }^{[3]}$. The methodology and approach are lack and pressed for. In this paper, the authors attempt to provide a description of framework for emergency rescue researches. 


\section{Emergency rescue concepts and characteristics}

\subsection{Emergency rescue concepts}

The trigger condition of emergency rescue action is the occurrence of disasters and crises. According to the Response Law on Emergency events of People's Republic of China issued and put in force in 2007, "emergency event" refers to "the bursting-out natural disasters, accident, public health incident and social security events that cause or could cause severe social damage and need to deal with by taking emergency measures."

Emergency rescue refers to all human intervention to cut down the emergency events and mitigate their damage in a broad sense, and protective, controllable and aid action to the individuals and key targets in a narrow sense. Emergency rescue mainly aims at natural disasters, accidents and public health incidents while the handling of which share similarities as centralized and unified actions could be taken in the aspect of human rescue, event control, public equipment and basic livelihood safeguard. Emergency danger-elimination work in emergency rescue is also known as rescue and relief work.

Emergency rescue is still a feeble and emerging interdisciplinary subject. The theoretical research on emergency rescue of emergency events which is pioneering, basic and strategic, not only involves the nurturing of emergency rescue commanding and technical personnel, but also bears great significance in promoting the building of national emergency system.

\subsection{The characteristics of emergency rescue operations}

Disasters and crises have the nature of the sudden urgency, complexity, severity, and decide the characteristics of emergency rescue operations are urgent, difficult, dangerous and hard.

\subsubsection{The situation is urgent}

The emergency events' occurrence and development mechanism is a nonlinear complex system, including a variety of natural and man-made factors intertwined. Even if you get a certain prediction early warning information, but disaster events' occurrence time, place, harm degree, consequences, are difficult to judge accurately. Disaster events occurred in recent years, showing a mass trend, the secondary events makes disaster events and derivative frequent more complex, resulting in harm to the rapid expansion and spread. After the burst of the emergency event if you do not respond promptly to their losses, over time, will be expanded dramatically, the situation is extremely urgent. For example, in Sichuan Wenchuan earthquake emergency rescue operations in 2008, the Tangjiashan quake lake was formed after a massive quake-triggered landslide from Tangjiashan Mountain blocked the Tongkou River, which ran through the Beichuan County, one of the worst-hit areas in the quake. The swollen lake is the largest of more than 30 quake lakes in Sichuan following the May 12 quake, posing a threat to 1.3 million people downstream. More than 250,000 people in low-lying areas in Mianyang have been relocated under a plan based on the assumption that a third of the lake volume breached its banks. The situation was urgent without delay.

\subsubsection{The action is difficult}

During the emergency rescue process, the natural environment and the human environment are often subjected to varying degrees of damage. Earthquakes, typhoons, floods, snowstorms and other major disasters lead a direct result: communication, transportation, electricity are basic interrupt even paralysis in the disaster area, seriously restricting the commencement of forces to organize and command and rescue operations. Major emergencies have integrated regional damage effects, the variety of the affected object to harmful levels of uneven performance of different type of disaster damage. Especially in the large-scale implementation of the rescue operation, the rescue force often dispersed configuration to multiple rescue operations of the operating area is facing some limitations. The technical equipment and professionals will be faced with the difficult to meet the real needs of limitations. Meanwhile, due to the long duration of the emergency rescue operations, as long as a few months, in the case of damage to the original social logistics system is affected by many factors interfere, rescue teams continued to self-supply more difficult.

\subsubsection{The Risk is intertwined}

The emergency rescue is a high-risk action, with the continued development of the emergency events, the secondary events and the derivative events continue to emerge, such as storms attacks, building collapse, burst dam failure, epidemic raging in storms and floods. The rescue work is facing a huge threat. For example, in the earthquake, relief and rescue teams 
faced three threats. First, the aftershocks threat, housing is in an unstable state after the earthquake, rescue operators facing with the threat of secondary collapse. The second threat is artificially collapsed. After buildings collapsed, although the collapsed body formed stable combination structure relatively, but the rescue operation is easy to destroy the original structure and loss of balance, trapped personnel and rescue workers face buried pressure is dangerous. The third threat is the continued events, the earthquake triggered fires, floods, explosions, toxic gas leak, a serious threat to the trapped workers, rescue also increase the risk.

\subsubsection{The task is hard}

Crisis event usually means facing serious political and international issues, if disposed of improperly, will evolve into a national disaster. In many countries, the army has the advantages of a high degree of combat readiness, well-organized, quick response and tenacious style, has become the choice for emergency rescue force, army image is an important part of national image building. The forest fires of Russia in the summer of 2010 exposed the existence of the problem of the Russian troops to participate in emergency rescue operations. Russian President Dmitry Medvedev inspected the burned of a naval base in the Moscow outskirts, quite ironically said: "The fire, I ask our armed forces to assume the important task of protecting people, unfortunately, some military departments cannot protect themselves". Affected by the fires, Russian government's support rate hit a low record. Thus, emergency rescue operations have special political significance.

An important indicator to examine emergency rescue operation in handling emergency events, especially in face of urgent, dangerous and difficult rescue task, is whether the response of professional forces is scientific and efficient.

\section{Framework of emergency rescue research}

The theoretic research framework on emergency rescue of emergency events includes: the organization and command of multi-forces of emergency rescue, the risk management and control of multi-disasters events, the standardization of rescue skills and equipments and professional training, and the information platform of emergency rescue (Fig. 1. ).

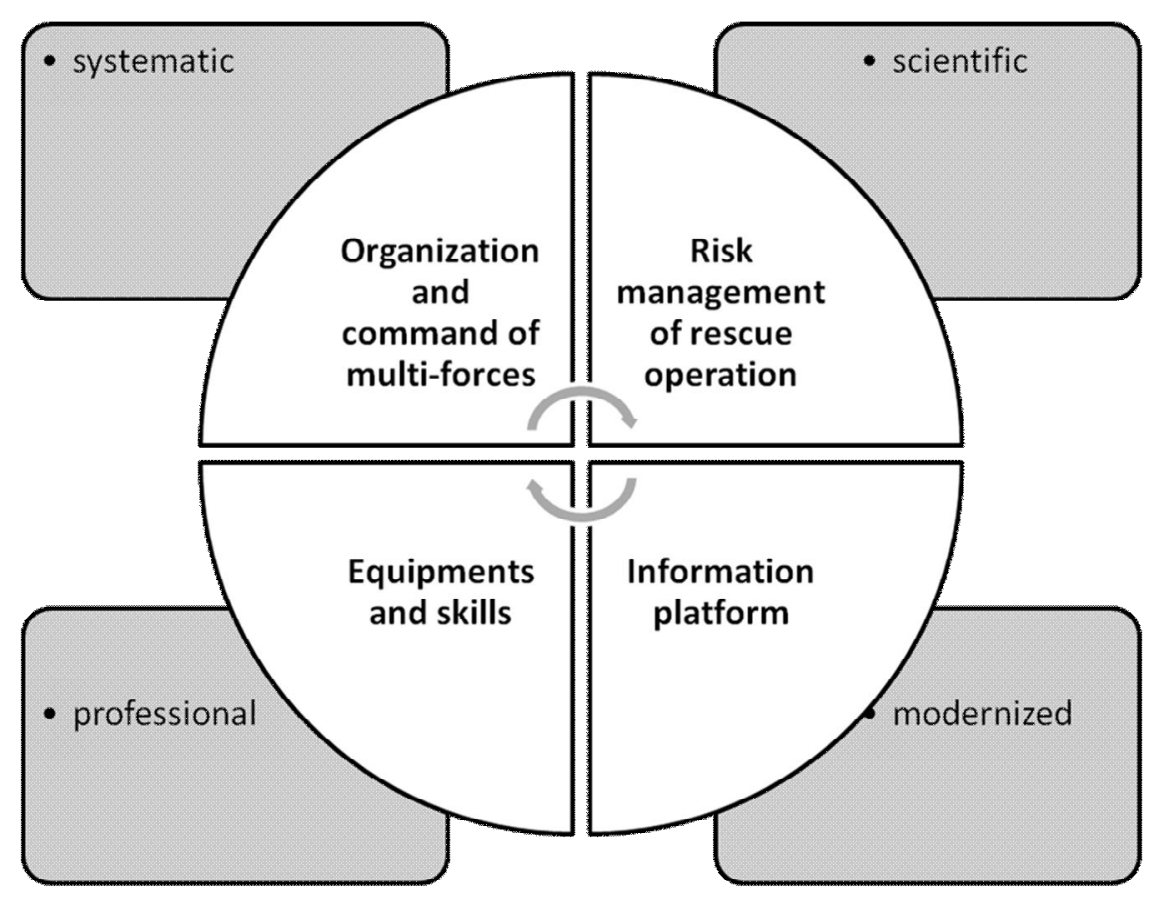

Fig. 1. The framework of emergency rescue research

\subsection{Organization and command of multi-forces}

"9.11" Event emergency rescue operation shows that coordination and communication were serious problems, and improvisation was common ${ }^{[4]}$. In
China, "5.12" earthquake emergency response actions sparked in-depth discussion for strategic delivery capability, professional force and so on.

The emergency rescue is a critical stage of emergency management. Its core is organization and 
command, including three levels of the strategic, operational and tactical.

Strategic level includes emergency rescue system, mechanism, and law. Its tasks are involved: research and judge the situation of emergency rescue in domestic and abroad emergency events; understand the frontier issues of emergency rescue, make the practical strategy for professional forces in emergency rescue; study the inner link among the leading system, coordination mechanism and relative regulations and laws, provide theoretical support in terms of perfecting system and mechanism for professional emergency rescue forces; investigate the key direction, basic layout and components in building emergency rescue forces, the vertical management and horizontal coordinative mechanism in rescue operation, as well as the involved rules, regulations and laws.

Operational level is joint command of multi-forces that includes: study the command structure and its components, levels, function and general conditions in on-spot joint command ${ }^{[5]}$; probe the coordination of professional forces and other forces, like public security and local governments on all-level, along with the organization and implementation of international cooperation in emergency rescue, in particular under the real situation of diversified emergency rescue forces.

Tactical level is on-spot command of emergency rescue. The following tasks should be done: study the on-spot command patterns based on the type of emergency events, software and hardware safeguard measures, the authorization and emergency decision on rescue operation, the quick and accurate delivery in special and sensitive regions, sealing and control of the spot, search and rescue of key targets, etc.

In organization and command of multi-force emergency rescue, being systematic is the focus.

\subsection{Risk management of rescue operation}

The role of the emergency rescue is to act immediately to prevent damage to life, property or environment ${ }^{[6]}$. Richard Yarwood considers the role of the emergency services in controlling, mitigating and resolving risk ${ }^{[7]}$.

The risk and evolution of emergency events involves: probe the evolutionary patterns of nurturing, happening, development and mutation of emergency events; get to know the types, tense, spatial and temporal distribution of the events by human intervention to stop the mutation for hazard, so as to provide direct scientific support to weaken the consequences.
The acceptor here means the objects which receive the actions produced or released or carried by the emergencies. We need to study the acceptor hazard-affected carrier, functional medium of the harmonious development of human society and natural environment, refers to the action object of emergency events, as well as the protective target of emergency rescue operation. The damage of hazard-affected carrier could lead to the activation or sudden release of its disaster elements, thereby causing secondary and derivative disasters and forming the chain of emergency events ${ }^{[3]}$. The key goal should be decided by studying different types of hazard-affected carriers so as to take proper action to block its happening and to achieve effective rescue.

Security management and control of emergency rescue is involved: study the security controlling technique in emergency rescue operation, master scientific methods, moment and dimension to measure and assess operation cost; mitigate the possible damage to the protective target and force staff by optimizing human intervention.

\subsection{Equipments and skills}

Equipment, technology and training are effective implementation of the basic condition of emergency rescue. The serialized system of emergency rescue equipments is necessary. The research content include the planning, design and application of serialized equipment for professional emergency rescue, command theory and method of equipment maintenance; research and develop new equipment as on-scene UAV (unmanned aerial vehicle) monitoring system in line with the series standards of equipment configuration based on various emergency rescue tasks.

On the basis of the equipment, study professional emergency rescue techniques of typical disasters as earthquake, geological incident, forest fire, flood and drought; learn the repairing technique of traffic facilities as highway, bridge, tunnel, airport and port, the maintenance technique of important highway and tunnels under special time and occasions, the levee engineering rescue technique, the dam reinforcement technique of reservoir and hydropower stations, the engineering danger-elimination technique of the dammed lake, the emergency repairing technique of converting station, transmission wire and oil and gas pipeline, as well as the rescue technique of helicopter. The retrospective review of all search and rescue (SAR) missions within Yosemite National Park during the 10-year study period between January 1990 and December 1999 shows, helicopter was the 
primary mode of transport in $28 \%$ of SAR incidents ${ }^{[8]}$.

To combine personnel, technology and equipment effectively, must be strengthened skill training of emergency rescue equipment. Probe the training methods, management, safeguard, patterns in view of base, analog, internet and situation, as well as interregional and joint training.

\subsection{Information platform}

Information is the basis for decision-making, the quality of information to determine the quality of the decision-making. Disasters and crises tend to disrupt the existing social order, resulting in distortion and confusion, affecting the quality of decision-making. Emergency rescue operations need to rely on the information management tools to ensure the smooth flow of information up and down. The fresh, accurate information is essential for effective response to large-scale emergencies. Information, at the top command level, at the field command level, and at the individual emergency personnel level, often difficult to obtain, process, interpret, and use for decision making in practice. So, we must strengthen the research of information hardware and software.

First, study the functional need of information collection, perception, positioning, delivery, summarization, research, distribution, integration and sharing; research, develop and integrate systematic modules as GIS (Geographic Information System), remote sensing and monitoring, professional model, analog emulation, command and control, aid decision making, comprehensive inquiry.

Second, database of emergency rescue cannot be ignored. The database consists of basic database, expert repository, case base of great emergency events, database of emergency events participated by professional rescue forces, model base of disaster prediction and pre-warning, scenario and plan base of emergency rescue operation.

We believe the high-tech but not make a perfect fetish of the high-tech, relying too much on high-tech to miscarriage of justice emergency rescue situation. We must give play to the initiative, and constantly strengthen the application research.

\section{Concluding comments}

In organizing and commanding, should focus on and improve top design. The emphasis should be laid on the systematic connection of strategy-battle-tactics level to produce a resultant force. In addition, Emergency rescue should be incorporated into the legal system, standardized and scientific track. In handling multiple types of emergency events, being scientific is the focus. The risk analysis and assessment in the whole process should be strengthened. The emphasis lays on the security management and control of responsive actions. In terms of equipments and skills of emergency rescue for professional teams, being professional is the focus. System and standard should be formed and implemented in specific training courses. As to the direction of informational construction of emergency rescue, being modernized is the focus. The specific functional need of application system and database should be clearly defined.

\section{Reference}

[1] C. C. Shan, On the construction of crisis management system with Chinese characteristics. China Executive Leadership Academy Pudong, 5:12-18, 2008.

[2] J.G. Liu, T. Dietz, S.R. Carpenter, et al. Complexity of coupled human and natural systems. AMBIO, 317 : 1513-1516, 2007.

[3] W. C. Fan, Y. Liu, W. G. Weng, Triangular Framework and "4+1" Methodology for Public Security Science and Technology. Science \& Technology Review, 6:3, 2009.

[4] B. Dearstyne, The FDNY on 9/11: Information and decision making in crisis. Government Information Quarterly, 24:29-46,2007.

[5] Z. Zhang, S. B. Zhong, H. Y. Yuan, The effective space theoretic model of the forest fire suppression and its actual battle analysis. Journal of Mountain Science, 5:534-539, 2010.

[6] Civil Contingencies Secretariat, Civil Contingencies Act 2004: A Short Guide. HMSO, London. 2004.

[7] R. Yarwood, Risk, rescue and emergency services: The changing spatialities of Mountain Rescue Teams in England and Wales. Geoforum, 41:257-270, 2010.

[8] K. Eric, M.D. Hung, A. David, et al. Search and rescue in Yosemite National Park: a 10-year review. Wilderness and Environmental Medicine, 18:111-116,2007. 